\section{IMMEDIATE EFFECT OF ARGENTINE TANGO PRACTICE ON PERSISTENT SYMPTOMS AND POSTURAL CONTROL DEFICITS ASSOCIATED WITH CONCUSSION}

Lise Worthen-Chaudhari, Catherine Quatman-Yates, W Jerry Mysiw, Eugenia Costa-Giomi, Ajit Chaudhari. The Ohio State University, Columbus, OH, USA

\subsection{6/bjsports-2021-IOC.120}

Background Concussion, or mild traumatic brain injury (mTBI), increases risk of brain and musculoskeletal injury after return to play (RTP). Dual-task training options have been suggested as a way to improve neurorehabilitation from concussion, thus reducing the risk of injury with RTP. To begin to evaluate the impact of dual task training on functional restoration, we report the immediate impact of an innovative dual task paradigm (Tango) on neurocognitive and neurosensorimotor symptoms.

Objective Assess within-session impact of Tango on symptoms and postural control.

Design

Repeated measures Immediate impact of Tango (pre v. post session; paired, 2-tailed Student's t-test)

Setting Community

Patients (or Participants) Adults with persistent concussion symptoms $3+$ months after date of injury.

Interventions (or Assessment of Risk Factors) One-to-one Adapted Argentine Tango dance lessons (Tango).

Main Outcome Measurements Primary Outcome Measure: Symptoms Score (Sports Concussion Assessment Tool v3). Secondary Outcome Measures: Postural sway (sway) during quiet standing with eyes closed (30 seconds minimum) measured through center of pressure (COP) calculations of resultant variability, velocity, and complexity (respectively: root-mean-square amplitude, mean velocity, and sample entropy calculated using the increment method).

Results Three participants referred from an Ohio State University concussion clinic underwent 16 one-to-one Tango sessions. When measured immediately before and after each Tango session, improvements were demonstrated in total symptoms score $(p=0.001)$ as well as sway variability $(p=0.007)$, velocity $(\mathrm{p}=0.013)$, and complexity $(\mathrm{p}=0.021)$. Per session, mean (SD) Tango dose to music per session was 24.2(5.2) minutes and Rating of Perceived Exertion was 9.2(1.4) (Borg scale, 6-20). Rating of Perceived Comfort during postural control testing with eyes closed was 1.1(0.3) (9 point scale, 1 high).

Conclusions Tango is feasible for adults with persistent concussion symptoms to engage in as a dual task activity and may help to improve persistent symptoms as well as postural control. More research is warranted regarding implications of this activity for supporting RTP.

\section{A CROSS-SECTIONAL STUDY OF LOW BACK PAIN AMONG RETIRED INTERNATIONAL ATHLETES (RUNNERS, SWIMMERS, ROWERS, AND HOCKEY PLAYERS)}

'Dale Cooper, ${ }^{2}$ Debbie Palmer, ${ }^{3}$ Mary O'Hanlon, ${ }^{4}$ Mark Batt. 'School of Allied Health Professions, University of Leicester, Leicester, UK; ${ }^{2}$ Institute for Sport, PE and Health Sciences, Moray House School of Education and Sport, University of Edinburgh, Edinburgh, UKi ${ }^{3}$ Academic Department of Orthopaedics, Trauma and Sports Medicine, University of Nottingham, Nottingham, UK; ${ }^{4}$ Centre for Sport, Exercise and Osteoarthritis Research Versus Arthritis, Queen's Medical Centre, Nottingham, UK

10.1136/bjsports-2021-IOC.121
Background Prevention of low back pain (LBP) requires the identification of modifiable risk factors. Currently there is limited evidence of these risk factors in current and retired athletic populations.

Objective To determine the prevalence and factors associated with LBP among retired international athletes (runners, swimmers, rowers, and hockey players).

Design Cross-sectional survey.

Patients (or Participants) 323 retired international level athletes, aged 30-97 years (median age 62 years), 43\% female $(n=$ 139) who had competed internationally for Great Britain.

Interventions (or Assessment of Risk Factors) Data was collected on age (years), sex, height $(\mathrm{cm})$, weight $(\mathrm{kg})$, sport and occupational history, bodily pain, training load, joint flexibility and medical history. A prior injury to the lumbar spine was defined by an injury causing pain $\geq 30$ days and requiring medical attention.

Main Outcome Measurements LBP (with or without leg pain) on most days of the past one-month.

Results Overall, the prevalence of LBP was $26.0 \%(84 / 323)$. The prevalence of LBP was 24.1\% (21/87) in rowers, $29.2 \%$ $(35 / 120)$ in runners, $31.3 \%(20 / 64)$ in swimmers, and $15.4 \%$ $(8 / 52)$ in hockey players. The odds ratio for LBP increased with a prior significant lumbar spine injury [OR 2.64; $95 \%$ CI, 1.43-4.89, $\mathrm{p}=0.002$ ], overweight BMI [OR 1.91; 95\% CI, 1.11-3.30, $\mathrm{p}=0.02]$, and was less prevalent among those aged 70 years and older [OR 2.37; 95\% CI, 1.02-5.54, p = 0.046]. No association was detected between LBP and female sex, a high training load, sporting discipline, heavy occupation post sports career, lumbar spine flexion, or comorbidities (i.e. diabetes, cancer, lung disease, stroke, heart disease).

Conclusions A prior significant lumbar spine injury and increased body mass index were associated with LBP in retired international athletes. Longitudinal follow-up is needed to determine if modification of these factors reduces the occurrence of LBP.

\section{LOW BACK PAIN AND ASSOCIATED FACTORS AMONG ITALIAN EQUESTRIAN ATHLETES: A CROSS-SECTIONAL STUDY}

${ }^{1,2}$ Mario Ferrante, ${ }^{3}$ Francesca Bonetti, ${ }^{2}$ Filippo Maria Quattrini, ${ }^{4}$ Maura Mezzetti ${ }^{5}$ Sabrina Demarie. ${ }^{1}$ Foro Italico' University Foundation, Rome, Italy; ${ }^{2}$ Medical Department of Italian Equestrian Sport Federation, Rome, Italy; ${ }^{3}$ Department of Clinical Sciences and Translational Medicine, University 'Tor Vergata', Rome, Italy: ${ }^{4}$ Department of Economics and Finance, University 'Tor Vergata', Rome, Italy; ${ }^{5}$ Department of Movement, Human and Health Sciences, University of Rome 'Foro Italico', Rome, Italy

\subsection{6/bjsports-2021-IOC.122}

Background Low back pain is a widespread condition in sport as in the general population.

Objective The purpose of this study is to investigate the prevalence and determinants of low back pain (LBP) and chronic low back pain (C-LBP) among Italian equestrian athletes Design Cross-sectional survey conducted using an online cloud-based software.

Setting Competitive athletes, members of the Italian Equestrian Sport Federation older than 18 years old $(n=40.932)$.

Participants 886 athletes participated in the survey: $21,90 \%$ were male and 78,10\% were female.

Assessment of Risk Factors Gender, age, weight, height, discipline practiced, years of practice, weekly training volume, athletic training were the independent variables considered. 